Whether auxetics are necessary for any form of cell-reproduction to occur is a point which will require further research to determine. It is, however, a striking fact that Dr. H. C. Ross was able for the first time to induce divisions in human leucocytes by means of auxetics, and was also able to demonstrate that the ova of Ascaris megalocephala will undergo division if incubated with auxetics. Dr. Fansham has also shown that Entamoeba coli can be caused to divide through many generations by means of these substances, whilst Dr. E. H. Ross has demonstrated that auxetics have a very remarkable action on trypanosomes.

From the foregoi $r$ facts it is clear that auxetics are a cause of cel. eproduction, and, although we cannot as vet state positively that there are no other causes, yet, judging from other biological examples, it is extremely probable that they are the sole cause, as it is very unlikely that a complex function like cellreproduction should have more than one direct cause. With regard to the presence of auxetics and linetics in pond water, I may say that I am at present investigating this point, and have definite proof of the presence, not onlv of auxetics, but also of kinetics or augmentors, in such waters. the latter bodies apparently varying according to the season, and also being dependent on the amount of albuminoid ammonia present.

Besides the presence of auxetics in hav infusion, there is one further point to be mentioned, viz. that if by the action of an enzyme, the cyst-wall in Colpoda were dissolved, quite enough auxetic would probably be liberated to cause division, were such necessary for development. This was well shown by Dr. H. C. Ross, who found that substances not themselves auxetics mav vet have auxetic action by causing limited cell death immediately within the walls of ova, thus setting free cnough auxetic to cause cell-division.

6) Ewhurst Road, Crofton Park, S.E. Aurrey H. Drew.

\section{Units of Pressure in Vacuum Work.}

Referring to the letter by Mr. Shaw in Nature of March 20 (p. 95), I beg leave to remind readers that we have already a convenient unit of pressure which, as fitting in an absolute system of units, is preferable to the micron of mercury, viz. the dyne per cm. ${ }^{2}$, or the barye of the c.g.s.-system. In fact, Prof. Knudsen has used it in all his later researches on molecular phenomena. In article V ro of the "Encvklopaedie der mathematischen Wissenschaften," p. 628, note is (Communications from the Physical Laboratory at Leyden, Supp1. No. 23, p. I4), by Prof. Kamerlingh Onnes and myself, we have given practically the same unit under the name of millitor as convenient for such pressures as those in Röntgen vacua.

In doing this we have followed the lead of the commission of the International Association of Refrigeration (Bull. de l'Ass. internat. du froid. 2, I9II, p. 38, rapporteur M. Ch. Ed. Guillaume). This commission proposed to accept the metre-kilogrammesecond system for general use, this one having better chances than the c.g.s.-system, and accordingly to introduce as an absolute unit of pressure the m.k.s.unit. As a practically identical realisation of it the commission proposed to introduce the international centitor (abbreviated for centi-torricelli), the international kilotor being equal to the pressure of a column of practically $75 \mathrm{~cm}$. of mercury under normal gravity (for further particulars see the article quoted above). Practically i millitor $=\mathrm{I} \mathrm{dvne} / \mathrm{cm}^{2}$, or barve, and within the accurary of experiments in the domain of these vacua I millitor $=0.75 \mu$ of mercury.

NO. 2268, VOL. 9I]
Seeing how simple this proportionality factor is, the work of reducing, say, McLeod gauge readings to millitors will not cause any appreciable trouble, whereas the indications by Prof. Knudsen in dynes $/ \mathrm{cm}^{2}$ are without any reduction expressed in millitors. For the highest vacua the microtor $=\mathrm{IO}^{-3}$ millitor $=$ $0.75 \times \mathrm{IO}^{-6} \mathrm{~mm}$. of mercury would be convenient. As abbreviations, mtor may be written for millitor. $\mu$ tor for microtor.

Physical Laboratory, Leyden.

\section{Reflection of X-Rays and X-Ray Fringes.}

Accounts of the reflection of X-rays and of $\mathrm{X}$-ray fringes contributed to Nature by Messrs. Bragg, Moseley, Barkla, Hupke, Keene, and others induce me to send you some results that I have obtained recently in the same direction.

I think that the appearances observed by Messrs. Laue, Friedrich, and Knipping are really due to the same cause as the reflected spots; they present quite a similar distribution and general character.

Fig. I shows the pattern obtained with a beam of Röntgen rays falling at an incidence of $80^{\circ}$ upon a face of a cube of rock-salt, the photographic plate being at right angles to the reflected beam. The reflected spots are similar to the transmitted spots, and present fringes perpendicular to the plane of inci-

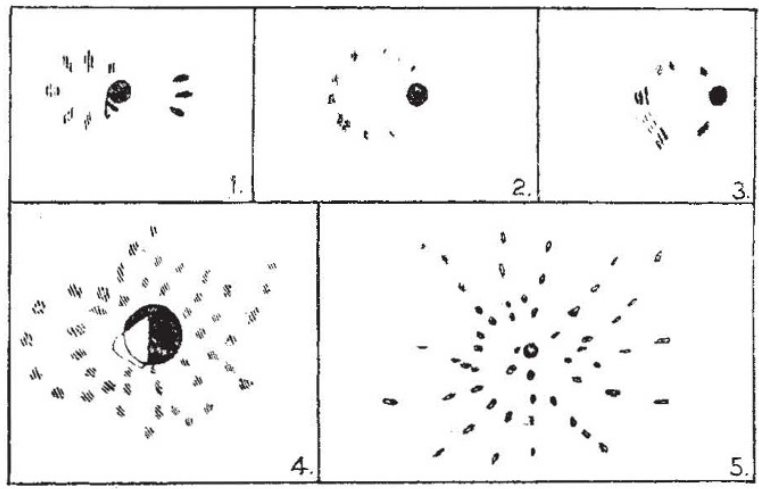

dence; they are situated on an elliptic curve, to which belongs also the point of impact of the primary beam. The spot on the main axis is regularly reflected; the others are symmetrically disposed, and possibly due to reflection on the planics of the corresponding octahedron, dodecahedron, \&c., which are in suitable positions. Fluorine gives the same pattern.

Fig. 2 is obtained with a beam (incidence $80^{\circ}$ ) falling upon the triangular face of an octahedric crystal of magnetite. The reflected spots show two systems of. fringes, one of which is approximately perpendicular to the plane of incidence.

It seems of importance to pay attention to the angle between the intersection of the plane of incidence and the quaternary axis situated in the cubic face of reflection. Fig. 3 shows that when this angle (which was o in Fig. I) becomes $45^{\circ}$, the curve of spots is deflected, the regularly reflected spot being no longer on the main axis but following the ordinary law of reflection; the distance of the fringes is probably slightly changed in this case.

I have often observed fringes in the transmitted spots obtained by Laue's methods. With octahedric magnetite (Fig. 4) all the spots (more than roo) were striated by parallel fringes; and on a plate (Fig. 5) obtained with fluorine the transmitted spots, the number of which was also very large, are all doubled in a radial direction. 